\title{
Poisson's equation and characterizations of reflexivity of Banach spaces
}

\author{
Vladimir P. Fonf, Michael Lin \\ Ben-Gurion University, Beer Sheva \\ E-mail: fonf@math.bgu.ac.il , lin@math.bgu.ac.il \\ Przemyslaw Wojtaszczyk \\ University of Warsaw \\ E-mail: wojtaszczyk@mimuw.edu.pl
}

\begin{abstract}
${ }^{1}$
Let $X$ be a Banach space with a basis. We prove that $X$ is reflexive if and only if every power-bounded linear operator $T$ satisfies Browder's equality

$$
\left\{x \in X: \sup _{n}\left\|\sum_{k=1}^{n} T^{k} x\right\|<\infty\right\}=(I-T) X
$$

We then obtain that $X$ (with a basis) is reflexive if and only if every strongly continuous bounded semi-group $\left\{T_{t}: t \geq 0\right\}$ with generator $A$ satisfies

$$
A X=\left\{x \in X: \sup _{s>0}\left\|\int_{0}^{s} T_{t} x d t\right\|<\infty\right\}
$$

The range $(I-T) X$ (respectively, $A X$ for continuous time) is the space of $x \in X$ for which Poisson's equation $(I-T) y=x(A y=x$ in continuous time) has a solution $y \in X$; the above equalities for the ranges express sufficent (and obviously necessary) conditions for solvability of Poisson's equation.
\end{abstract}

\section{INTRODUCTION}

Let $X$ be a (real or complex) Banach space. Poisson's equation (which was originally for the Laplacian in certain function spaces) has been abstracted to solving the equation $A y=x$ for a given $x \in X$, where $A$ is the infinitesimal generator of a strongly continuous one-parameter bounded semi-group of linear operators $\left\{T_{t}: t \geq 0\right\}$ (see [9]).

In "discrete time", Poisson's equation for a power-bounded linear operator $T$ is the solution of $(I-T) y=x$ for a given $x \in X$. In ergodic theory, elements of $(I-T) X$ are called coboundaries, and it is of interest to find conditions for $x$ to be a coboundary, i.e. for the solvability of Poisson's equation.

Obviously, since $\left\|\frac{1}{n} \sum_{k=1}^{n} T^{k} x\right\| \rightarrow 0$ if and only if $x \in \overline{(I-T) X}$ (e.g. [8]), for any power-bounded $T$ on $X$ we have

$$
(I-T) X \subset\left\{x \in X: \sup _{n}\left\|\sum_{k=1}^{n} T^{k} x\right\|<\infty\right\} \subset \overline{(I-T) X}
$$

\footnotetext{
${ }^{1}{ }_{2000}$ Mathematics Subject Classification: Primary 46B10, 47A35; Secondary 47D06
} 
It was proved by F. Browder [2] (and rediscovered in [3]) that if $X$ is reflexive, then for every $T$ power-bounded on $X$ we have

$$
(I-T) X=\left\{x \in X: \sup _{n}\left\|\sum_{k=1}^{n} T^{k} x\right\|<\infty\right\}
$$

Browder's equality (1) means that a solution $y$ to Poisson's equation $(I-T) y=x$ exists if (and only if) $\sup _{n}\left\|\sum_{k=1}^{n} T^{k} x\right\|<\infty$.

In this paper we prove that if $X$ is a Banach space with a basis such that (1) holds for every power-bounded $T$ on $X$, then $X$ is reflexive. The continuous time analogue of this result is then deduced in $\S 4$.

A bounded linear operator $T$ on a (real or complex) Banach space $X$ is called mean ergodic if

$$
E(T) x:=\lim _{n \rightarrow \infty} \frac{1}{n} \sum_{k=1}^{n} T^{k} x \quad \text { exists } \quad \forall x \in X .
$$

The general mean ergodic theorem, proved (independently) by Lorch, by Kakutani and by Yosida, says that if $X$ is a reflexive Banach space, then every power-bounded linear operator $T$ is mean ergodic (see [8]). In [5] we proved that if $X$ is a Banach space with a basis, then mean ergodicity of all power-bounded operators implies reflexivity of $X$.

For $T$ power-bounded, mean ergodicity is equivalent to the ergodic decomposition $X=F(T) \oplus \overline{(I-T) X}$, where $F(T)$ is the space of fixed points of $T$. In [11] it was shown that if $(I-T) X$ is closed (without assuming mean ergodicity), then $T$ is mean ergodic, and $\left\|\frac{1}{n} \sum_{k=1}^{n} T^{k}-E(T)\right\| \rightarrow 0$ (i.e. $T$ is uniformly ergodic).

In the sequel we denote $S(T):=\left\{x \in X: \sup _{n}\left\|\sum_{k=1}^{n} T^{k} x\right\|<\infty\right\}$. It was shown in [4] that $S(T)$ is closed if and only if $(I-T) X$ is closed, which is equivalent to uniform ergodicity of $T$. If $X$ is infinite-dimensional and has a basis, then by [5, Corollary 3] it has a power-bounded $T$ which is not uniformly ergodic, so in general $S(T)$ is not closed.

Browder's equality (1) was proved in [12] for every contraction of $L_{1}(\mu)$ (and in [1] for certain power-bounded operators of $L_{1}$ ), so this equality in general does not imply mean ergodicity. This result of [12] also shows that having (1) for every contraction is not sufficient to obtain reflexivity; see [6] for an example of a nonreflexive $X$ with a basis and separable dual, such that all contractions of $X$ and all contractions of $X^{*}$ are mean ergodic and satisfy (1).

\section{Preliminary Results}

Although our first result follows from our main theorem, it follows also from [5], and its proof leads to some conditions for mean ergodicity. 
Theorem 2.1. The following are equivalent for a Banach space $X$ :

(i) $X$ is reflexive.

(ii) every power-bounded operator $T$ defined on a closed subspace $Y \subset X$ satisfies

$$
(I-T) Y=\left\{y \in Y: \sup _{n}\left\|\sum_{k=1}^{n} T^{k} y\right\|<\infty\right\}
$$

(iii) every mean ergodic power-bounded operator $T$ defined on a closed subspace $Y \subset X$ satisfies (2).

Proof. Assume first that $X$ is reflexive. Then any closed subspace $Y$ is reflexive, and for $T$ power-bounded on a reflexive Banach space $Y$ the equality (2) follows from $[2]$.

Clearly (ii) implies (iii).

Assume now that $X$ is not reflexive. By the ergodic characterization of [5], there exists a closed subspace $Z$ and a power-bounded operator $S$ on $Z$ which is not mean ergodic. Take $z \in Z$ such that $\frac{1}{n} \sum_{k=1}^{n} S^{k} z$ does not converge, and put $y_{0}:=(I-S) z$. Define $Y=\overline{(I-S) Z}$; then $Y$ is $S$-invariant, and we put $T:=S_{\mid Y}$. Clearly $\sup _{n}\left\|\sum_{k=1}^{n} T^{k} y_{0}\right\|<\infty$, which yields $\left\|\frac{1}{n} \sum_{k=1}^{n} T^{k} y_{0}\right\| \rightarrow 0$. Вy the definitions $\left\|\frac{1}{n} \sum_{k=1}^{n} T^{k} y\right\| \rightarrow 0$ for any $y \in Y$, so $\overline{(I-T) Y}=Y$.

If $T$ (defined on $Y$ ) satisfies (2), then there exists $y_{1} \in Y$ with $y_{0}=(I-T) y_{1}$. We then have $(I-S)\left(z-y_{1}\right)=(I-S) z-(I-T) y_{1}=0$, which yields

$$
z-y_{1}=\frac{1}{n} \sum_{k=1}^{n} S^{k}\left(z-y_{1}\right)=\frac{1}{n} \sum_{k=1}^{n} S^{k} z-\frac{1}{n} \sum_{k=1}^{n} T^{k} y_{1} .
$$

Since $\left\|\frac{1}{n} \sum_{k=1}^{n} T^{k} y_{1}\right\| \rightarrow 0$, the above yields $\frac{1}{n} \sum_{k=1}^{n} S^{k} z \rightarrow z-y_{1}$, contradicting the choice of $z$. Hence the mean ergodic operator $T$ on $Y$ does not satisfy (2).

For any power-bounded $T$ on a Banach space $X$ we have

$$
(I-T) \overline{(I-T) X} \subset(I-T) X \subset\left\{x \in X: \sup _{n}\left\|\sum_{k=1}^{n} T^{k} x\right\|<\infty\right\}
$$

Equality in the second inclusion does not imply mean ergodicity - equality holds for every contraction $T$ on $L_{1}$, even not mean ergodic [12]. The operator $T$ constructed in the proof of Theorem 2.1 is mean ergodic, but there is no equality in the second inclusion above.

Proposition 2.2. A power-bounded operator $T$ on a Banach space $X$ is mean ergodic if (and only if) $(I-T) \overline{(I-T) X}=(I-T) X$.

Proof. If $T$ is mean ergodic, then $X=F(T) \oplus \overline{(I-T) X}$, and the condition follows. 
Assume that $T$ is not mean ergodic. We apply the proof of Theorem 2.1 with $Z=X$, in which case $Y=\overline{(I-T) X}$, and obtain $y_{0}$ which is in $(I-T) X \subset\{y \in Y$ : $\left.\sup _{n}\left\|\sum_{k=1}^{n} T^{k} y\right\|<\infty\right\}$ but is not in $(I-T) Y$, hence $(I-T) Y \neq(I-T) X$.

Theorem 2.3. Let $X$ be a Banach space with a basis. $X$ is reflexive if and only if every power-bounded operator $T$ on $X$ satisfies

$$
\left\{x \in X: \sup _{n}\left\|\sum_{k=1}^{n} T^{k} x\right\|<\infty\right\}=(I-T) \overline{(I-T) X}
$$

Proof. If $X$ is reflexive, then every power-bounded $T$ is mean ergodic, so we have $(I-T) \overline{(I-T) X}=(I-T) X$, and (4) holds by applying (1) to $T$.

Assume now that a power-bounded $T$ on $X$ satisfies (4). Then by (3) we have $(I-T) \overline{(I-T) X}=(I-T) X$, and thus $T$ is mean ergodic by Proposition 2.2. If every power-bounded $T$ satisifes (4), then $X$ is reflexive by the characterization in [5] for Banach spaces with a basis.

Theorem 2.4. Let $T$ be power-bounded on a Banach space $X$. If $\overline{(I-T) X}$ is reflexive, then $T$ is mean ergodic, and Browder's equality (1) holds.

Proof. Since $Y:=\overline{(I-T) X}$ is reflexive and $T$-invariant, by [2] we have $\{y \in$ $\left.Y: \sup _{n}\left\|\sum_{k=1}^{n} T^{k} y\right\|<\infty\right\}=(I-T) Y$. If $T$ is not mean ergodic, the proof of Theorem 2.1 with $Z=X$ yields $(I-T) Y \neq\left\{y \in Y: \sup _{n}\left\|\sum_{k=1}^{n} T^{k} y\right\|<\infty\right\}$, a contradiction. The mean ergodicity of $T$ yields that $X=F(T) \oplus Y$, and thus

$$
(I-T) X=(I-T) Y=\left\{y \in Y: \sup _{n}\left\|\sum_{k=1}^{n} T^{k} y\right\|<\infty\right\}
$$

Since $\sup _{n}\left\|\sum_{k=1}^{n} T^{k} x\right\|<\infty$ implies $x \in Y$, (1) holds and the theorem is proved.

Remark. Reflexivity of $\overline{(I-T) X}$ is far from being necessary for mean ergodicity of $T$.

\section{THE MAIN RESULT}

In view of (3), equality (4) implies (1), and our main result below improves Theorem 2.3. It provides an improvement of Theorem 2.1 when $X$ has a basis.

Theorem 3.1. The following are equivalent for a (separable) Banach space $X$ with a basis:

(i) $X$ is reflexive.

(ii) every power-bounded T on $X$ satisfies Browder's equality (1).

(iii) every mean ergodic power-bounded $T$ on $X$ satisfies (1). 
When $X$ is reflexive, all power-bounded operators $T$ satisfy (1) by [2], so we have to show only (iii) implies (i).

It was proved in [4, Theorem 2.3] that a power-bounded operator $T$ in a Banach space $X$ satisfies (1) if and only if $(I-T) X$ is an $F_{\sigma}$-set in $X$. To prove the theorem, we follow the strategy of [5]. If $X$ is non-reflexive and has a basis, then by [13] it has a non-shrinking basis. Therefore Theorem 3.1 is a consequence of the following.

Theorem 3.2. Let $X$ be a Banach space having a non-shrinking finite-dimensional Schauder decomposition. Then there exists a power-bounded mean ergodic linear operator $T$ such that $(I-T) X$ is not an $F_{\sigma}$-set.

The first step is the following lemma of [5].

Lemma 3.3. Let $X$ be a Banach space with a non-shrinking Schauder decomposition. Then $X$ has a Schauder decomposition $X=\sum_{k} X_{k}$ with the following property: there exist a functional $h \in X^{*}$ and a sequence $\left\{e_{k}\right\}$ such that for every $k \geq 1$ we have $e_{k} \in X_{k},\left\|e_{k}\right\| \leq 1$ and $h\left(e_{k}\right)=1$.

Furthermore, if the components of the original non-shrinking decomposition are finite-dimensional, so are all the $X_{k}$.

The last part of the lemma follows from the construction in [5] - each $X_{k}$ is a finite sum of components of the original decomposition.

As noted at the beginning of the proof of [5, Theorem 1], we can change the norm to an equivalent one so that in the decomposition obtained in the above lemma the coordinate projections $Q_{k}: X \longrightarrow X_{k}$ and the partial sums projections $P_{k}: X \longrightarrow \sum_{j=1}^{k} X_{j}$ (defined respectively by $Q_{k}\left(\sum_{j=1}^{\infty} x_{j}\right)=x_{k}$ and $\left.P_{k}=\sum_{j=1}^{j} Q_{k}\right)$ have norm 1 .

Lemma 3.4. Let $X=\sum_{k} X_{k}$ be the Schauder decomposition, with coordinate projections $Q_{k}$, obtained in lemma 3.3, let $e_{0}=0$, and put $u_{n}=e_{n}-e_{n-1}$ for $n \geq 1$. For $k \geq 1$ define $E_{2 k}=\operatorname{span}\left\{u_{k}\right\}$ and $E_{2 k-1}=X_{k} \bigcap$ ker $h$. Then $X=\sum_{m} E_{m}$ is a Schauder decomposition of $X$, with coordinate projections $\bar{Q}_{m}$ given by

$\bar{Q}_{2 k-1}=R_{k} Q_{k}$, where $R_{k}: X_{k} \longrightarrow E_{2 k-1}$ is defined by $R_{k} x_{k}=x_{k}-h\left(x_{k}\right) e_{k}$.

$\bar{Q}_{2 k} x=\left(h-\sum_{j=0}^{k-1} Q_{j}^{*} h\right)(x) u_{k}$, where $Q_{0}=0$.

Proof. For $x \in X_{k}$ we have $x-h(x) e_{k} \in E_{2 k-1}$, and $\sum_{j=1}^{k} u_{j}=e_{k}$. Hence $\sum_{m=1}^{2 n} E_{m}=\sum_{k=1} X_{k}$, and $\operatorname{span}\left\{\cup_{m} E_{m}\right\}$ is dense in $X$.

We first show that each $\bar{Q}_{m}$ as defined is a projection onto $E_{m}$ which vanishes on $E_{l}$ for $l \neq m$.

It is easily checked that $R_{k}$ is a projection of $X_{k}$ onto $E_{2 k-1}$, for any $k \geq 1$, so $R_{k} Q_{k} R_{k} Q_{k}=R_{k} R_{k} Q_{k}=R_{k} Q_{k}$, and thus $\bar{Q}_{2 k-1}$ is a projection onto $E_{2 k-1}$. Since $Q_{k} X_{j}=\{0\}$ for $j \neq k$, we have $\bar{Q}_{2 k-1} E_{2 j-1}=\{0\}$ for $j \neq k$.

Since $u_{l} \in X_{l-1} \oplus X_{l}$, we have $Q_{k} E_{2 l}=\{0\}$ when $k<l-1$ or $k>l$. For $l=k$ we have $Q_{k} u_{l}=e_{k}$ and $R_{k} Q_{k} u_{l}=R_{k} e_{k}=0$ since $h\left(e_{k}\right)=1$. For $l=k+1$ we have $Q_{k} u_{l}=-e_{k}$ and $R_{k} Q_{k} u_{l}=0$. Thus $\bar{Q}_{2 k-1} E_{m}=\{0\}$ for $m \neq 2 k-1$. 
We now look at $\bar{Q}_{2 k}$. By definition it takes $X$ into $E_{2 k}$, so to show it is a projection it is enough to check that $\bar{Q}_{2 k} u_{k}=u_{k}$. We compute

$$
\bar{Q}_{2 k} u_{k}=\left(h\left(u_{k}\right)-\sum_{j=0}^{k-1} h\left(Q_{j} u_{k}\right)\right) u_{k}=
$$

$\left(h\left(e_{k}\right)-h\left(e_{k-1}\right)-h\left(Q_{k-1} u_{k}\right)\right) u_{k}=\left(h\left(e_{k}\right)-h\left(e_{k-1}\right)+h\left(e_{k-1}\right)\right) u_{k}=h\left(e_{k}\right) u_{k}=u_{k}$.

For $x \in E_{2 l-1}$ we have $h(x)=0$, and $Q_{j} x=0$ for $j \neq l, h\left(Q_{l} x\right)=h(x)=0$. Hence $\bar{Q}_{2 k} E_{2 l-1}=\{0\}$.

For $k=1$ we have $\bar{Q}_{2} x=h(x) u_{1}=h(x) e_{1}$ so for $l>1$ we obtain $\bar{Q}_{2} u_{l}=$ $h\left(u_{l}\right) u_{1}=0$. For $k>1$ and $l \neq k$ we have

$$
\bar{Q}_{2 k} u_{l}=\left(h\left(u_{l}\right)-\sum_{j=1}^{k-1} h\left(Q_{j} u_{l}\right)\right) u_{k}=\left(h\left(e_{l}\right)-h\left(e_{l-1}\right)-\sum_{j=1}^{k-1}\left[h\left(Q_{j} e_{l}\right)-h\left(Q_{j} e_{l-1}\right]\right) u_{k} .\right.
$$

This is 0 for $l>k$ since in the sum all terms are 0 . For $l \leq k-1$ we have in the sum only $h\left(e_{l}\right)-h\left(e_{l-1}\right)=0$, so $\bar{Q}_{2 k} u_{l}=0$ for $l \neq k$.

We thus have that each $\bar{Q}_{m}$ is a projection onto $E_{m}$ with $\bar{Q}_{m} E_{j}=\{0\}$ for $j \neq m$. This yields also that $E_{m} \cap E_{j}=\{0\}$ for $j \neq m$.

Claim: Put $\bar{P}_{n}=\sum_{j=1}^{n} \bar{Q}_{j}$. Then $\sup _{n}\left\|\bar{P}_{n}\right\|<\infty$.

We denote $P_{n}=\sum_{j=1}^{n} Q_{j}$. Since $\left\{X_{n}\right\}$ is a Schauder decomposition of $X$, we have $\sup _{n}\left\|P_{n}\right\|<\infty$.

Fix $n$ and let $m>n$. Using $Q_{j} x=R_{j} Q_{j} x+h\left(Q_{j} x\right) e_{j}$, for $x \in \sum_{k=1}^{m} X_{k}$ we obtain

$$
\begin{gathered}
\bar{P}_{2 n} x=\sum_{j=1}^{2 n} \bar{Q}_{j} x=\sum_{k=1}^{n} R_{k} Q_{k} x+\sum_{k=1}^{n}\left(h(x)-\sum_{j=0}^{k-1} h\left(Q_{j} x\right)\right)\left(e_{k}-e_{k-1}\right)= \\
\sum_{k=1}^{n} R_{k} Q_{k} x+\sum_{j=0}^{n-1} h\left(Q_{j} x\right) e_{j}+\left(h(x)-\sum_{j=0}^{n-1} h\left(Q_{j} x\right)\right) e_{n}= \\
\left.\sum_{k=1}^{n} Q_{k} x+\left(h(x)-\sum_{j=0}^{n} h\left(Q_{j} x\right)\right) e_{n}=P_{n} x+\left(h-\sum_{j=0}^{n} Q_{j}^{*} h\right)(x)\right) e_{n}=P_{n} x+\left(h-P_{n}^{*} h\right)(x) e_{n} .
\end{gathered}
$$

Since $\left\|e_{n}\right\|=1$, we obtain $\left\|\bar{P}_{2 n} x\right\| \leq\left\|P_{n}\right\| \cdot\|x\|+\left\|I-P_{n}^{*}\right\| \cdot\|h\| \cdot\|x\|$, so $\sup _{n}\left\|\bar{P}_{2 n}\right\| \leq$ $\sup _{n}\left\|P_{n}\right\|+\|h\|\left(1+\sup _{n}\left\|P_{n}\right\|\right)$.

We now have $\bar{P}_{2 n+1}=\bar{P}_{2 n}+\bar{Q}_{2 n+1}$, so the above yields

$$
\bar{P}_{2 n+1}=P_{n} x+\left(h-P_{n}^{*} h\right)(x) e_{n}+R_{n+1} Q_{n+1} x
$$

But $\left\|R_{n+1} Q_{n+1} x\right\| \leq\left\|Q_{n+1} x\right\|+\|h\| \cdot\left\|Q_{n+1} x\right\|$, and $\sup _{n}\left\|Q_{n}\right\|<\infty$, so we obtain $\sup _{n}\left\|\bar{P}_{2 n+1}\right\|<\infty$, and the claim is proved.

Since $\lim \bar{P}_{m} x=x$ on a dense subset, the claim yields that $\bar{P}_{m} x \rightarrow x$ on all of $X$ and $\sum_{m=1}^{\infty} E_{m}$ is a Schauder decomposition. 
Proposition 3.5. Let $X=\sum_{k} X_{k}$ be a Schauder decomposition of $X$ with coordinate projections $Q_{k}$. For a sequence $a:=\left\{a_{j}\right\}_{j=1}^{\infty}$ with $a_{j}>0$ for $j \geq 1$ and $\sum_{j=1}^{\infty} a_{j}=1$ put $A_{k}=\sum_{j=1}^{k} a_{j}$. Then for every $x \in X$ the series $\sum_{k=1}^{\infty} A_{k} Q_{k} x$ converges in norm, and the operator $T_{a} x:=\sum_{k=1}^{\infty} A_{k} Q_{k} x$ is power-bounded on $X$.

Proof. The proposition follows from the computations on pages 150-151 of [5] (with $h=0)$. In these computations it is assumed that the coordinate projections $Q_{k}$ and the partial sums $P_{k}=\sum_{j=1}^{k} Q_{j}$ all have norm 1 (and then $\sup _{n}\left\|T_{a}^{n}\right\| \leq 2$ ); the assumption is achieved by a change to an equivalent norm.

Proof of Theorem 3.2: Let $X=\sum_{k=1}^{\infty} E_{k}$ be the Schauder decomposition of $X$ obtained in Lemma 3.4 from the non-shrinking Schauder decomposition $X=$ $\sum_{k} X_{k}$ with finite-dimensional components. By the definition, all the $E_{k}$ are finitedimensional, and let $\bar{Q}_{k}$ be the coordinate projection on $E_{k}$.

Choose $a=\left\{a_{j}\right\}_{j=1}^{\infty}$ with $a_{j}>0$ and $\sum_{j=1}^{\infty} a_{j}=1$, such that the tails $b_{k}=$ $\sum_{j=k+1}^{\infty} a_{j}$ satisfy $\sum_{k=1}^{\infty} b_{k}<\infty$ (e.g. $\left.a_{j}=\frac{1}{2^{j}}\right)$, and put $T x=T_{a} x=\sum_{k=1}^{\infty} A_{k} \bar{Q}_{k} x$. By the proposition above, $T$ is power-bounded. By the definitions $(I-T) x=$ $\sum_{m=1}^{\infty} b_{m} \bar{Q}_{m} x$, so $I-T$ is a compact operator since $E_{m}$ are finite-dimensional.

We assert that $(I-T) X$ is not an $F_{\sigma}$-set. We prove this by contradiction - we assume that $(I-T) X$ is an $F_{\sigma}$-set.

By the construction in Lemma 3.4, the sequence $\left\{\sum_{i=1}^{n} u_{i}\right\}_{n \geq 1}$ is bounded, so compactness of $I-T$ implies that there is a subsequence $\left\{n_{p}\right\}$ with $(I-T) e_{n_{p}}=$ $(I-T)\left(\sum_{i=1}^{n_{p}} u_{i}\right) \rightarrow z$. Since $(I-T) X$ is an $F_{\sigma}$, by [4, Theorem 2.3] the unit ball $U$ of $X$ satisfies $\overline{(I-T) U} \subset(I-T) X$, so $z \in(I-T) X$. Let $x_{0} \in X$ satisfy $(I-T) x_{0}=z$.

Claim: $x_{0}=\sum_{i=1}^{\infty} \alpha_{i} u_{i}$

The claim means that $\bar{Q}_{2 k-1} x_{0}=0$ for every $k \geq 1$. Fix $k$ and denote $m=2 k-1$. If $\bar{Q}_{m} x_{0} \neq 0$, then there exists $f \in X^{*}$ with

$$
\bar{Q}_{m}^{*} f\left(x_{0}\right)=f\left(\bar{Q}_{m} x_{0}\right)=\left\|\bar{Q}_{m} x_{0}\right\|>0 .
$$

Since $(I-T)^{*} \bar{Q}_{m}^{*} f=\sum_{j=1}^{\infty} b_{j} \bar{Q}_{j}^{*} \bar{Q}_{m}^{*} f=b_{m} \bar{Q}_{m}^{*} f$, we obtain $\bar{Q}_{m}^{*} f=\frac{1}{b_{m}}(I-T)^{*} \bar{Q}_{m}^{*} f$, SO

$$
\begin{gathered}
\left\|\bar{Q}_{m} x_{0}\right\|=\bar{Q}_{m}^{*} f\left(x_{0}\right)=\frac{1}{b_{m}}(I-T)^{*} \bar{Q}_{m}^{*} f\left(x_{0}\right)=\frac{1}{b_{m}} \bar{Q}_{m}^{*} f\left((I-T) x_{0}\right)=\frac{1}{b_{m}} \bar{Q}_{m}^{*} f(z)= \\
\frac{1}{b_{m}}\left(\bar{Q}_{m}^{*} f\right)\left(\lim _{p \rightarrow \infty}(I-T) \sum_{i=1}^{n_{p}} u_{i}\right)=\frac{1}{b_{m}} \lim _{p \rightarrow \infty}\left((I-T)^{*} \bar{Q}_{m}^{*} f\right)\left(\sum_{i=1}^{n_{p}} u_{i}\right)= \\
\lim _{p \rightarrow \infty} \bar{Q}_{m}^{*} f\left(\sum_{i=1}^{n_{p}} u_{i}\right)=\lim _{p \rightarrow \infty} f\left(Q_{2 k-1} \sum_{i=1}^{n_{p}} u_{i}\right)=0
\end{gathered}
$$

contradicting the assumption $\bar{Q}_{2 k-1} x_{0} \neq 0$. This proves the claim. 
The sequence $\left\{u_{n}\right\}$ is obviously a basic sequence (basis for $\sum_{k>1} E_{2 k}$ ), and by the computation of $\bar{Q}_{2 k}$ in Lemma 3.4, its biorthogonal sequence is $u_{n}^{*}=h-\sum_{j=0}^{n-1} Q_{j}^{*} h$. For $x \in E_{2 k-1}=X_{k} \cap \operatorname{ker} h$ we have $h(x)=0$ and $Q_{j} x=0$ for $j \neq k$, so $u_{n}^{*}(x)=$ $h(x)-\sum_{j=0}^{n-1} h\left(Q_{j} x\right)=0$ since the sum is 0 for $n \leq k$ and $h\left(Q_{k} x\right)=h(x)=0$ for $n>k$. By the definition of $T$ we have

$$
\begin{aligned}
& (I-T)^{*} u_{n}^{*}(x)=u_{n}^{*}((I-T) x)=u_{n}^{*}\left(\sum_{m=1}^{\infty} b_{m} \bar{Q}_{m} x\right)= \\
& b_{2 n} u_{n}^{*}\left(\bar{Q}_{2 n} x\right)+\sum_{k=1}^{\infty} b_{2 k-1} u_{n}^{*}\left(\bar{Q}_{2 k-1} x\right)=b_{2 n} u_{n}^{*}\left(\bar{Q}_{2 n} x\right) .
\end{aligned}
$$

We now use the claim and the biorthogonality to obtain

$$
\begin{gathered}
u_{k}^{*}\left(x_{0}\right)=u_{k}^{*}\left(\bar{Q}_{2 k} x_{0}\right)=\frac{1}{b_{2 k}}(I-T)^{*} u_{k}^{*}\left(x_{0}\right)=\frac{1}{b_{2 k}} u_{k}^{*}\left((I-T) x_{0}\right)= \\
\frac{1}{b_{2 k}} u_{k}^{*}(z)=\frac{1}{b_{2 k}} \lim _{p \rightarrow \infty} u_{k}^{*}\left((I-T) \sum_{i=1}^{n_{p}} u_{i}\right)=\lim _{p \rightarrow \infty} u_{k}^{*}\left(\sum_{i=1}^{n_{p}} u_{i}\right)=1
\end{gathered}
$$

using the $T$-invariance of the $E_{m}$. But this is a contradiction, since $u_{k}^{*}\left(x_{0}\right)=$ $h\left(x_{0}-\sum_{j=0}^{k-1} Q_{j} x_{0}\right) \rightarrow 0$. Hence $(I-T) X$ is not an $F_{\sigma}$-set.

Finally, since each component $E_{m}$ is $T$-invariant and finite-dimensional, $T$ is mean ergodic on each component, and therefore, since $T$ is power-bounded on $X$, it is mean ergodic. This proves Theorem 3.2.

\section{On POISSON'S EqUATION FOR ONE-PARAMETER SEMI-GROUPS}

Originally, Poisson's equation was for the Laplacian. This has been abstracted to solving the equation $A y=x$ for a given $x \in X$, where $A$ is the infinitesimal generator of a strongly continuous one-parameter bounded semi-group of linear operators $\left\{T_{t}: t \geq 0\right\}$ (see [9]). We use Theorem 3.1 to obtain a characterization of reflexivity by a condition for solvability of Poisson's equation, for all infinitesimal generators of bounded strongly continuous semi-groups.

Theorem 4.1. The following are equivalent for a Banach space $X$ with a basis:

(i) $X$ is reflexive.

(ii) Every strongly continuous bounded semi-group $\left\{T_{t}: t \geq 0\right\}$ with generator $A$ satisfies

$$
A X=\left\{x \in X: \sup _{s>0}\left\|\int_{0}^{s} T_{t} x d t\right\|<\infty\right\}
$$

(iii) Every uniformly continuous bounded semi-group $\left\{T_{t}: t \geq 0\right\}$ with generator A satisfies (5). 
Proof. (i) implies (ii) by Theorem 2.6 of [9] (since the dual semi-group is also strongly continuous, by reflexivity and [7, Theorem 10.6.5]).

Obviously (ii) implies (iii). We show that (iii) implies (i).

Assume that $X$ (with a basis) is not reflexive. By Theorem 3.1 there exists a power-bounded operator $T$ such that (1) fails, which means that for some $x \notin$ $(I-T) X$ we have $\sup _{n}\left\|\sum_{k=1}^{n} T^{k} x\right\|<\infty$. We may assume, by changing the norm to an equivalent one, that $\|T\|=1$. For $t \geq 0$ put $S_{t}=e^{t(T-I)}$. Then $\left\{S_{t}\right\}$ is a uniformly continuous semigroup, with infinitesimal generator $A=T-I$. The power series expansion yields

$$
\left\|S_{t}\right\|=e^{-t}\left\|e^{t T}\right\| \leq e^{-t} e^{t\|T\|}=1 .
$$

Since $\sup _{n}\left\|\sum_{k=1}^{n} T^{k} x\right\|<\infty$, Theorem 5 of [12] yields the existence of some $y^{* *} \in$ $X^{* *}$ such that $\left(I-T^{* *}\right) y^{* *}=x$; hence $x \in A^{* *} X^{* *}$ (we have identified $X$ with its canonical image in $\left.X^{* *}\right)$. The uniform continuity of $\left\{S_{t}\right\}$ implies that of $\left\{S_{t}^{* *}\right\}$, with generator $A^{* *}=T^{* *}-I$, and for $s>0$ we obtain

$$
\left\|\int_{0}^{s} S_{t} x d t\right\|=\left\|\int_{0}^{s} S_{t}^{* *} x d t\right\|=\left\|-S_{s}^{* *} y^{* *}+y^{* *}\right\| \leq 2\left\|y^{* *}\right\| .
$$

Since $x \notin(I-T) X=A X$, the contraction semi-group $\left\{S_{t}\right\}$ does not satisfy (5). Hence $X$ is reflexive when (iii) holds.

Remark. The idea of using the semi-group $e^{t(T-I)}$ is due to Rainer Nagel, in the context of characterizing reflexivity by mean ergodicity of all bounded semi-groups $[10]$.

Acknowledgements. Parts of this research were carried out during visits of the first two authors to the Institute of Mathematics of the Polish Academy of Sciences (IMPAN) in Warsaw, supported respectively by EU programs SPADE and TODEQ, and during a visit of the third author to Ben-Gurion University, supported by its Center for Advanced Studies in Mathematics. The authors are grateful for the hospitality of their hosts. The third author was also partially supported by the Polish Ministry of Science and Higher Education grant N201 269335.

\section{REFERENCES}

[1] I. Assani, A note on the equation $Y=(I-T) X$ in $L^{1}$, Illinois J. math. 43 (1999), 540-541.

[2] F. Browder, On iteration of transformations in non-compact minimal dynamical systems, Proc. Amer. Math. Soc. 9 (1958), 773-780.

[3] P. Butzer and U. Westphal, The mean ergodic theorem and saturation, Indiana Univ. Math. J. $20(1970 / 71), 1163-1174$.

[4] V. Fonf, M. Lin and A. Rubinov, On the uniform ergodic theorem in Banach spaces that do not contain duals, Studia Math. 121 (1996), 67-85.

[5] V. Fonf, M. Lin and P. Wojtaszczyk, Ergodic characterizations of reflexivity of Banach spaces, J. Functional Anal. 187 (2001), 146-162. 
[6] V. Fonf, M. Lin and P. Wojtaszczyk, A non-reflexive Banach space with all contractions mean ergodic, Israel J. Math., to appear.

[7] E. Hille and R. Phillips, Functional analysis and semi-groups, American Math. Soc., Providence, RI, 1957.

[8] U. Krengel, Ergodic theorems, De Gruyter, Berlin, 1985.

[9] U. Krengel and M. Lin, On the range of the generator of a Markovian semigroup, Math. Z. 185 (1984), 553-565.

[10] D. Mugnolo, A semigroup analogue of the Fonf-Lin-Wojtaszczyk ergodic characterization of reflexivity of Banach spaces with a basis, Studia Math. 164 (2004), 243-251.

[11] M. Lin, On the uniform ergodic theorem, Proc. Amer. Math. Soc. 43 (1974), 337-340.

[12] M. Lin and R. Sine, Ergodic theory and the functional equation $(I-T) x=y$, J. Operator theory 10 (1983), 153-166.

[13] M. Zippin, A remark on bases and reflexivity in Banach spaces, Israel J. Math. 6 (1968), 74-79. 